\title{
Correction to: Nonlinear Circuits and Systems with Memristors
}

\section{Correction to:}

\section{F. Corinto et al., Nonlinear Circuits and Systems} \section{with Memristors,}

https://doi.org/10.1007/978-3-030-55651-8

The original version of this book was inadvertently published without updating author corrections in Abstract for the following Chapters 1, 2, 3, 4, 5, 6, 7, 8, 9, 10 , and 11. Now, the corrections have been incorporated in all the chapter abstracts in Online.

The original version of this book inadvertently published with an error in affiliation of Dr. Mauro Forti in Online. Now, the affiliation has been corrected in Online. 